\title{
Static Output Feedback Control for Lane Change Maneuver
}

\author{
Saïd Mammar, Hichem Arioui
}

\begin{abstract}
This paper addresses the lateral control of a vehicle during lane change maneuvers. The proposed design procedure aims to answer the questions of control using cost-effective sensors implementation, adaptation to measured variables and robustness to unmeasured varying parameters. This is achieved through a static output feedback controller with preview information. The only used measurements are the lateral displacement at sensor location and the yaw angle relative to the lane centerline. The vehicle lateral model is augmented with an integral action, the error signal and the preview reference signal. The controller is synthesized using the LMI framework thanks to a relaxation method that removes the nonlinear terms. Simulations are conducted for various scenarios showing the ability of the design method to handle different performance objectives.
\end{abstract}

\section{INTRODUCTION}

Several research works have addressed autonomous lane keeping maneuver during the last thirty years. Several control methods have been applied ranging from classical PID controller to advanced robust and nonlinear approaches. Presently, several market vehicles are already equipped with lane keeping systems working mainly in nominal driving conditions. However, complete vehicle automation needs also lane changing maneuver handling in order to allow more flexible use of the lane capacity and to ensure that the vehicle can for example take hand over the driver in order to avoid a collision by changing lane.

Performing automatic lane change maneuver will really provide an advance in driving comfort and safety [1]. Lane change involves both longitudinal and lateral dynamics on one side and vehicle positioning on the road and relative to others vehicles. In contrast, autonomous lane change maneuver has been less addressed. Works mainly focus on traffic conditions allowing performing the maneuver and on path planning computation allowing to design a trajectory for the vehicle from a present lane to the adjacent one. In [4] the question on when and how to perform a lane change maeneuver has been examined. Determining whether a lane change maneuver is possible results from a reachability analysis problem [3]. Maneuver generation is addressed in [5] and [19]. When an appropriate traffic gap is available with a corresponding time instance, the manoeuver is initiated by controlling the longitudinal and the lateral motion. Lane change maneuver has been addressed as a kinematic

S. Mammar and H. Arioui are with Evry Val d'Essonne University, Université Paris-Saclay, France. IBISC: Informatique, Biologie Intégrative et Systèmes Complexes - EA 4526, 40 rue du Pelvoux CE1455, 91020, Evry, Cedex, France, (e-mail: (said.mammar, hichem.arioui) @ibisc.univevry.fr).

This work has been conducted as a part of Université Paris-Saclay research project INVASHIVE. trajectory planning and control problems solved using Model Predictive Control [7], [8]. Various research studies have previously applied MPC for trajectory planning and motion control of automated vehicles [9],[10]. Other control methods have been used to control the trajectory following in the inner-loop. Among controller design method, loopshaping robust $\mathrm{H}_{\infty}$ control has been applied in [11]. An $\mathrm{H}_{\infty}$ controller which combines a closed-loop component with a prefilter controller which achieves robust model reference tracking has been introduced in [12]. A fuzzy controller has been considered in [13]. Lane change maneuver has also been treated in the situation of cooperation with human driver [14] and [15]. Performing the maneuver in adverse conditions has been considered in [16] and [17]. In [18] control aspects during emergency lane change maneuver have been proposed. Further results concerning lane change maneuver for automated highways are provided in [19], [20], [21] including receding horizon control approaches.

In this paper, the aim is to design a very simple implementable controller. An output feedback controller overcomes the necessity to have access to the whole state vector and avoids the use of observers in order to estimate it. Dynamic output feedback has been considered under different control objectives, which are converted into Linear Matrix Inequalities (LMI) conditions [23] and [24]. Static output feedback is more difficult to design under LMI framework. It has been considered in [25] and [26] by introducing a transformation matrix about the system output matrices. Further improvements have been proposed in [27], [28] and [29] addressing different classes of systems. Even if the design of static output feedback controller is more complicated, it offers a simple interpretation of the controller action as it directly maps the measurements to the control input.

In addition, controllers are generally designed in order to ensure stabilization of the system. However, we generally intend that the output tracks a given reference signal which is normally known in advance. A simple way to enhance controller performance for trajectory tracking is to include preview actions taking into account the terms of the reference trajectory for feedback [30], [32] and [34]. The preview range is adjustable and directly impacts the performance of the system.

This paper proposes the design of a static output feedback controller which includes preview terms of the lane change reference trajectory. The controller uses the only available measurements, namely the lateral displacement and the relative yaw rate. The vehicle lateral model is put under a polytopic form allowing to take into account the variations of the forward speed as a varying parameter [28] and [31]. 
This leads to a system with four submodels. In addition, an integrator is added to the system in order to ensure zeros steady state error for constant road curvature. Afterwards, the preview information of reference signal to be tracked is included in the model state-space vector. A static output feedback controller is thus synthesized using Linear Matrix inequalities (LMI) framework. This controller feedbacks the error signal, the measurement and the reference signal. The controller is tested under several conditions which highlight the performances of the proposed approach.

The remainder of the paper is organized as follows. Section II is dedicated for the polytopic vehicle lateral model development. The control objectives are also detailed in this section. Section III focusses of the system adaptation for the controller design. The controller is shown to result from the solution of an LMI problem in which some parameters are priorly chosen. Section IV considers some simulation tests under different conditions and discusses some practical design adjustments. Finally section $\mathrm{V}$ provides some conclusions and future works.

\section{VEHICLE LATERAL MODEL}

The vehicle model is based on the classical bicycle model for lateral dynamics and the lane positioning dynamics [22]. In this model, the left and right lateral forces are projected on virtual wheels located on the vehicle longitudinal axle at a distance $l_{f}$ from the center of gravity for the front one and at a distance $l_{r}$ from the center of gravity for the rear one (Figure 1).

The model includes the lateral dynamics (Figure 1). The front and rear lateral forces, $F_{y f}$ and $F_{y r}$ respectively, are directly related to the front sideslip angle $\alpha_{f}$ and rear sideslip angle $\alpha_{r}$ respectively. These forces start with a linear region allowing the approximations $F_{y f}=C_{f} \alpha_{f}$ and $F_{y r}=C_{r} \alpha_{r}$ respectively, before saturation. The coefficients $C_{f}$ and $C_{r}$ are the front and rear tire cornering stiffness respectively.

The model state variables are: the sideslip angle at the center of gravity $\beta$ which is related to the forward and the lateral velocities $v_{y}=v_{x} \beta$, the yaw rate $\dot{\psi}$, the relative yaw angle $\psi_{L}$ and the lateral displacement $y_{L}$ as state variables. The later is considered to be measured at a preview distance $l_{s}$. The preview distance is naturally constrained by the sensor range and the vehicle environment. The look forward measurement provides the system with a lead phase. The optimal value of $l_{s}$ can be determined according to the forward speed.

The control input of this model is the tire steering angle $\delta_{f}$ while the road curvature $\rho_{\text {ref }}$ is considered as a disturbance input. The model state-space equations are:

$\dot{x}(t)=A\left(v_{x}, \frac{1}{v_{x}}\right) x(t)+B\left(\frac{1}{v_{x}}\right) \delta_{f}(t)+E\left(v_{x}\right) \rho_{r e f}(t)$
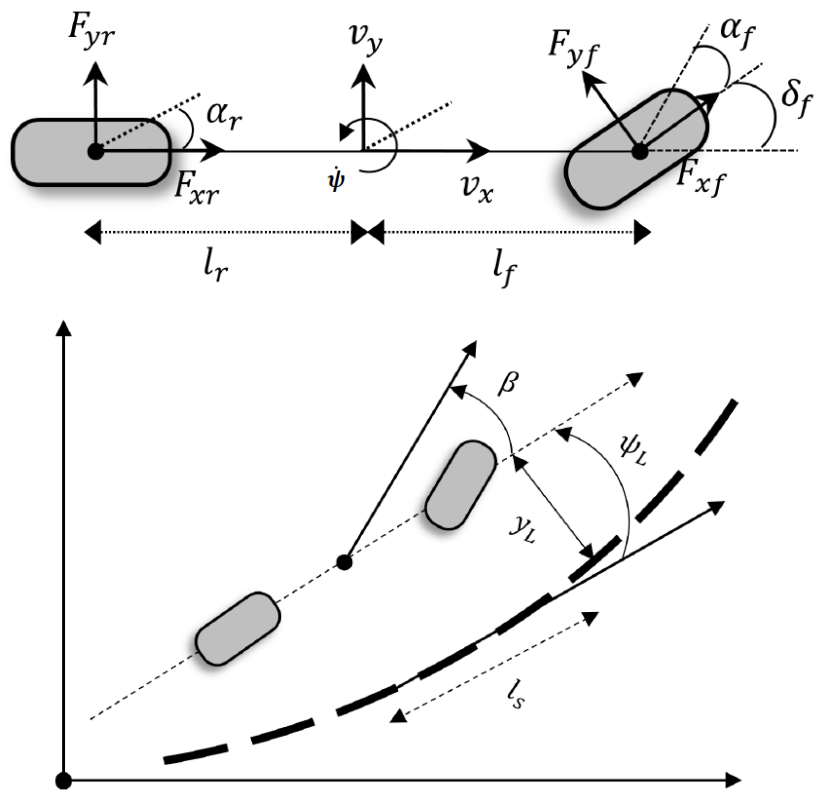

Fig. 1. Lateral dynamics and positionning

where

$$
\begin{aligned}
A & =\left[\begin{array}{cccc}
a_{11} & a_{12} & 0 & 0 \\
a_{21} & a_{22} & 0 & 0 \\
0 & v_{x} & 0 & 0 \\
1 & l_{s} v_{x} & v_{x} & 0
\end{array}\right], \\
B^{T} & =\left[\begin{array}{llll}
b_{1} & b_{2} & 0 & 0
\end{array}\right], \\
E^{T} & =\left[\begin{array}{llll}
0 & 0 & -v_{x} & -l_{s} v_{x}
\end{array}\right],
\end{aligned}
$$

and

$$
\begin{array}{ll}
a_{11}=-\frac{C_{f}+C_{r}}{m v_{x}}, & a_{12}=\frac{C_{r} l_{r}-C_{f} l_{f}}{m v_{x}}-v_{x}, \\
a_{21}=\frac{-C_{f} l_{f}+C_{r} l_{r}}{J}, & a_{22}=-\frac{C_{f} l_{f}^{2}+C_{r} l_{r}^{2}}{J}, \\
b_{1}=\frac{C_{f}}{m v_{x}}, & b_{2}=\frac{C_{f} l_{f}}{J} .
\end{array}
$$

The state vector is $x=\left[\beta, \frac{\dot{\psi}}{v_{x}}, \psi_{L}, y_{L}\right]^{T}$. Notice that the second state-space variable is chosen as $\frac{r}{v_{x}}$ in order to remove the dependency on $\frac{1}{v_{x}^{2}}$ of the second term of the first row of the $A$ matrix. The numerical values of the above parameters are given in Table 1 in the appendix. In the following, it is assumed that only $y_{L}$ and $\psi_{L}$ are measured and thus available for feedback. The control input is the front steering angle.

\section{A. Takagi-Sugeno model}

Model of equation (1) exhibits terms depending on the forward speed $v_{x}$ and its inverse $\frac{1}{v_{x}}$. The speed appears thus linearly and nonlinearly. The speed is assumed to be controlled by another loop and is measured. The Takagi-Sugeno (TS) modelling framework, allows to describe the parameter dependent model under a set of submodels depending on the extremal values of the parameters. This representation is called nonlinear sector approximation [35]. In fact, defining 
$\rho_{1}=v_{x}$ and $\rho_{2}=\frac{1}{v_{x}}$, a four submodels TS system is achieved

$$
\dot{x}(t)=\sum_{i=1}^{4} \theta_{i}\left(\bar{A}_{i} x(t)+\bar{B}_{i} \delta_{f}(t)+\bar{E}_{i} \rho_{\text {ref }}(t)\right)
$$

where $\theta_{i} \geq 0,1 \leq i \leq 4, \sum_{i=1}^{4} \theta_{i}=1$ and

$$
\begin{aligned}
& \theta_{1}=m_{11} m_{21}, \quad \theta_{2}=m_{11} m_{22} \\
& \theta_{3}=m_{12} m_{21}, \quad \theta_{4}=m_{12} m_{22}
\end{aligned}
$$

with

$$
\begin{aligned}
& m_{11}=\frac{v_{M}-\rho_{1}}{v_{M}-v_{m}}, \quad m_{12}=1-m_{11} \\
& m_{21}=\frac{\frac{1}{v_{m}}-\rho_{2}}{\frac{1}{v_{m}}-\frac{1}{v_{M}}}=\frac{v_{M}-v_{M} v_{m} \rho_{2}}{v_{M}-v_{m}}, \quad m_{22}=1-m_{21}
\end{aligned}
$$

The matrices $\left(\bar{A}_{i}, \bar{B}_{i}, \bar{E}_{i}\right), 1 \leq i \leq 4$ are obtained from

$$
\begin{aligned}
\left(\bar{A}_{1}, \bar{B}_{1}, \bar{E}_{1}\right) & =\left(A\left(v_{m}, \frac{1}{v_{M}}\right), B\left(\frac{1}{v_{M}}\right), E\left(v_{m}\right)\right) \\
\left(\bar{A}_{2}, \bar{B}_{2}, \bar{E}_{2}\right) & =\left(A\left(v_{m}, \frac{1}{v_{m}}\right), B\left(\frac{1}{v_{m}}\right), E\left(v_{m}\right)\right) \\
\left(\bar{A}_{3}, \bar{B}_{3}, \bar{E}_{3}\right) & =\left(A\left(v_{M}, \frac{1}{v_{M}}\right), B\left(\frac{1}{v_{M}}\right), E\left(v_{M}\right)\right) \\
\left(\bar{A}_{4}, \bar{B}_{4}, \bar{E}_{4}\right) & =\left(A\left(v_{M}, \frac{1}{v_{m}}\right), B\left(\frac{1}{v_{m}}\right), E\left(v_{M}\right)\right)
\end{aligned}
$$

Notice that the obtained model is an exact representation of the model of equation (1) in the compact set $v_{m} \leq$ $v_{x} \leq v_{M}$. The obtained model is a continuous time model. However for implementation aspects and when considering preview information, it is more convenient to consider it in discrete time domain. In fact the preview information is only available at some sensor sample times. Knowing that, the model is discretized using a simple Euler method leading to the discrete-time state-space model with sample time of $T=0.05 \mathrm{sec}$.

$\left\{\begin{array}{l}x(k+1)=\sum_{i=1}^{4} \theta_{i}\left(A_{i} x(k)+B_{i} \delta_{f}(k)+E_{i} \rho_{\text {ref }}(k)\right) \\ y(k)=C x(k)\end{array}\right.$

where $\left(A_{i}, B_{i}, C_{i}\right)=\left(I_{4}+T \bar{A}_{i}, T \bar{B}_{i}, T \bar{E}_{i}\right)$.

\section{CONTROLler DESIGN}

In the following, the road curvature is considered constant. Taking the difference operator on both sides of equation (3) leads to:

$$
\left\{\begin{array}{l}
\Delta x(k+1)=\sum_{i=1}^{4} \theta_{i}\left(A_{i} \Delta x(k)+B_{i} \Delta \delta_{f}(k)\right) \\
\Delta y(k)=C \Delta x(k)
\end{array}\right.
$$

Notice that since the road curvature is considered as constant, taking the difference operator cancels it from the equations. In addition, the integral input which is added at the control input will ensure a zeros steady state error on circular bends.

Let us now define the error signal $e(k)$ as the difference between the output $y(k)$ and the reference signal $r(k)$ to be tracked, that is

$$
e(k)=y(k)-r(k)
$$

Let us now define the augmented plant with the augmented state vector $\tilde{x}=\left[e^{T}(k), \Delta x^{T}(k)\right]^{T}$. The augmented statespace system reads

$$
\left\{\begin{array}{l}
\tilde{x}(k+1)=\sum_{i=1}^{4} \theta_{i}\left(\tilde{A}_{i} \tilde{x}(k)+\tilde{B}_{i} \Delta \delta_{f}(k)+G_{p} \Delta r(k)\right) \\
e(k)=\tilde{C} \tilde{x}(k)
\end{array}\right.
$$

where

$$
\begin{aligned}
& \tilde{A}_{i}=\left[\begin{array}{cc}
I_{2} & C \\
0 & A_{i}
\end{array}\right], \tilde{B}_{i}=\left[\begin{array}{c}
0 \\
B_{i}
\end{array}\right], \\
& G_{p}=\left[\begin{array}{c}
-I_{2} \\
0
\end{array}\right], \tilde{C}=\left[\begin{array}{ll}
I_{2} & 0
\end{array}\right] .
\end{aligned}
$$

Suppose now that the reference signal values are known $n_{p}$ samples ahead. Let us define the vector

$$
x_{r}(k)=\left[\begin{array}{lll}
\Delta r^{T}(k) & \ldots & \Delta r^{T}\left(k+n_{p}\right)
\end{array}\right]^{T}
$$

and the matrix

$$
A_{r}=\left[\begin{array}{ccccc}
0 & I_{2} & 0 & \cdots & 0 \\
0 & 0 & \ddots & \ddots & \vdots \\
\vdots & \vdots & \ddots & \ddots & 0 \\
0 & \cdots & \cdots & 0 & I_{2} \\
0 & \cdots & \ldots & 0 & 0
\end{array}\right]
$$

The objective now is to embed the reference signal appearing in equation (6) into a state vector. This is achieved by defining the state vector

$$
\hat{x}(k)=\left[\begin{array}{ll}
\tilde{x}^{T}(k) & x_{r}^{T}(k)
\end{array}\right]^{T}
$$

which allows to obtain the augmented system

$$
\left\{\begin{array}{l}
\hat{x}(k+1)=\sum_{i=1}^{4} \theta_{i}\left(\hat{A}_{i} \hat{x}(k)+\hat{B}_{i} \Delta \delta_{f}(k)\right) \\
y_{p}(k)=\hat{C} \hat{x}(k)
\end{array}\right.
$$

where

$$
\begin{gathered}
\hat{A}_{i}=\left[\begin{array}{cc}
\tilde{A}_{i} & G_{p} \\
0 & A_{r}
\end{array}\right], \hat{B}_{i}=\left[\begin{array}{c}
\tilde{B}_{i} \\
0
\end{array}\right], \\
\hat{C}=\left[\begin{array}{ccc}
I_{2} & & \\
& C & \\
& & I_{\left(n_{p}+1\right) \times q}
\end{array}\right] .
\end{gathered}
$$

The aim now is to design a static output feedback controller of the form

$$
\Delta u(k)=\Delta \delta_{f}(k)=\left(\sum_{i=1}^{4} \theta_{i} K_{i}\right) y_{p}(k)
$$

This controller combines four gains each of them dedicated for one of the submodels. In fact, the real-time availability of the parameter $\theta_{i}$ allows to adapt the gain values.

When incorporating controller (8) into the augmented system (7), one easily obtain the closed-loop system:

$$
\hat{x}(k+1)=\sum_{i=1}^{4} \theta_{i}\left(\hat{A}_{i}+\hat{B}_{i}\left(\sum_{j=1}^{4} \theta_{j} K_{j}\right) \hat{C}\right) \hat{x}(k)
$$


The gains $K_{j}$, $\left.(1 \leq j \leq 4)\right)$, have to be computed such that the system (9) is asymptotically stable, which means $\lim _{k \rightarrow+\infty} \hat{x}(k)=0$ and as the error $e(k)$ is a part of the state $\hat{x}(k)$, it is ensured also that $\lim _{k \rightarrow+\infty} e(k)=0$.

Designing the matrix gains $K_{j}$ is not simple to handle using LMI techniques due on one hand to the inherent difficulty to design a static output feedback and on the other hand to the crossing terms product $\hat{B}_{i} K_{j}$. In fact the synthesis of a static output feedback is primarily a bilinear matrix inequality problem (BMI). Several approaches have been developed which involve equality constraints. Relaxation method has been proposed for example in [33].

This paper adapts the main result presented in [34]. It allows to introduce relaxation terms and parameter dependent matrices $G(\theta)$ and $P(\theta)$ defined as:

$$
G(\theta)=\sum_{i=1}^{4} \theta_{i} G_{i}, \quad P(\theta)=\sum_{i=1}^{4} \theta_{i} P_{i}
$$

Following the results provided in [34], given a positive scalar $\nu$ and matrices $Q, W$ of compatible dimensions, if there exist matrices $P_{i}>0, U_{i}, L_{i}, G_{i}, 1 \leq i \leq 4$ of compatible dimensions such that

$$
\Pi_{i j}+\Pi_{j i}<0 \quad(1 \leq i \leq j \leq 4)
$$

where

$$
\Pi_{i j}=\left(\begin{array}{ccc}
-G_{i}-G_{j}^{T}+P_{i} & * & * \\
\hat{A}_{i} G_{j}+\hat{B}_{i} L_{j} Q & -P_{i} & * \\
\hat{C} G_{j}-U Q & \nu W^{T} L_{i}^{T} \hat{B}_{j}^{T} & \Theta
\end{array}\right)
$$

and

$$
\Theta=-\nu U W-\nu W^{T} U^{T},
$$

then the system (9) is robustly asymptotically stable, and the gain matrix can be obtained by

$$
K_{i}=L_{i} U^{-1}
$$

One can notice that this matrix gain involves the following components

$$
K_{i}=\left(\begin{array}{lllll}
K_{e i} & K_{y i} & K_{r i}(0) & \ldots & K_{r i}\left(n_{p}\right)
\end{array}\right)
$$

thus the command increment is given by

$$
\Delta u(k)=K_{e} e(k)+K_{y} \Delta y(k)+\sum_{j=0}^{n_{p}} K_{r}(j) \Delta r(k+j)
$$

where

$$
\begin{aligned}
& K_{e}=\sum_{i=1}^{4} \theta_{i} K_{e i}, \quad K_{y}=\sum_{i=1}^{4} \theta_{i} K_{y i}, \\
& K_{r}(j)=\sum_{i=1}^{4} \theta_{i} K_{r i}(j)
\end{aligned}
$$

and finally the control input is obtained from

$$
u(k)=\delta_{f}(k)=K_{e} \sum_{j=0}^{k} e(k)+K_{y} y(k)+\sum_{j=0}^{n_{p}} K_{r}(j) r(k+j)
$$

\section{Simulation RESUlts}

The vehicle speed range is considered varies from $v_{m}=$ $10 \mathrm{~m} / \mathrm{s}$ to $v_{M}=25 \mathrm{~m} / \mathrm{s}$. The controller is designed is the following parameters $\nu=0.1, Q=0.6 \hat{C}, W=0.2 \hat{C}^{T} \hat{C}$. The controller gains are computed from solving the LMI (12).

\section{A. Lane change on straight road}

The lane change maneuver is first performed on a straight road and it is initiated at time $t=1 \mathrm{sec}$. The sample time is set to $T=0.05 \mathrm{sec}$. The reference signal is constituted of 5 samples with incremental values of $0.6 \mathrm{~m}, 1.2 \mathrm{~m}, 1.8 \mathrm{~m}$ and $2.4 m$ then reaching the value of $3 m$ which corresponds to the position of the adjacent lane. Its profile is depicted in Figure 2. After a time duration of $10 \mathrm{sec}$, the vehicle is driven back automatically to the initial lane, simulating thus a kind of obstacle avoidance maneuver.

The system responses with the preview controller are shown in solid lines while those without the preview action are given in dashed lines in Figure 2. The final reference value is reached within 5 steps of $(0.2,0.4,0.6,0.8,1) \times 3 m$. The needed steering angle is depicted in the second plot of the same figure. The lateral velocity and the yaw rate are provided in the third and the fourth plot of figure 2, while the relative yaw angle and the lateral displacement are shown in the fifth and sixth plot of the same figure. The steering angle is smooth, and the lateral velocity is limited and is within comfort limits as it is less than $2 \mathrm{~m} / \mathrm{s}$. The maneuver is performed within $3 \mathrm{sec}$ with very limited overshot on the lateral displacement (Figure 2) the reference signal is tracked without steady state error.

Comparing the solid and dashed lines of the Figure 2 shows the advantages in using the preview information for control as using the same design procedure but without the preview controller leads to much slower responses provided in dashed lines.
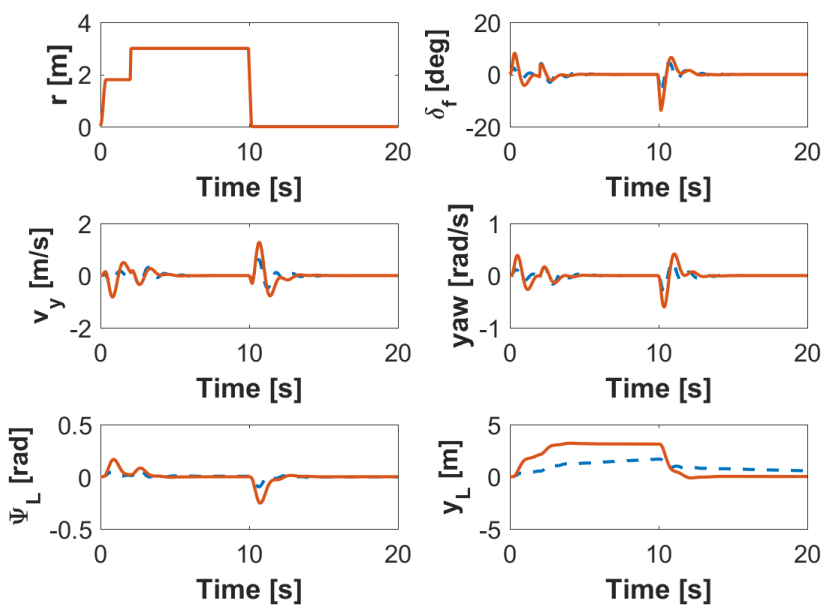

Fig. 2. Reference signal and steering angle 


\section{B. Lane change on curved road}

The lane change maneuver is now considered on a curved road with a curvature of $\rho_{\text {ref }}=1 / 250 \mathrm{~m}^{-1}$. Entering the bend is initiated after an autonomous lane change maneuver of $3 \mathrm{sec}$. At the end of this time duration, the bend starts and enters the system as a constant step input. Results are depicted in Figure 3. As the curvature acts as a perturbation, its effect is visible on the plots of the different state space variables which have non zero steady-state values. These value correspond to the stationary ones on a circular bend. Even if the responses exhibit overshot during the transient phase, the performances are still good as all the requirements are achieved.
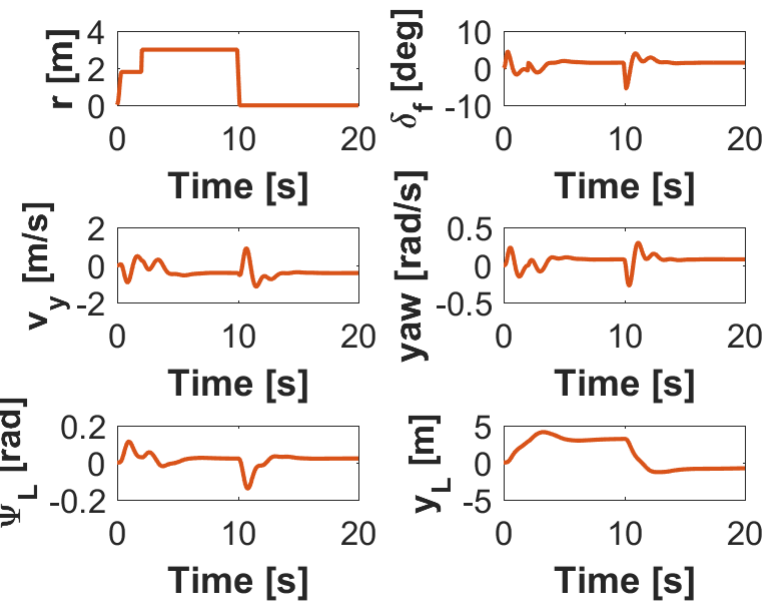

Fig. 3. Lane change maneuver on curved road

\section{Lane change on low friction road}

Road friction is subject to variations however its value is not available for measurement. Even if it is not possible to make the controller adaptive to these variations, it is still possible to compute the controller for the polytopic system constituted by the submodels obtained at the extreme values of the road adhesion stiffness $c_{f}$ and $c_{r}$. These coefficients appear as linear parameters in the state-space matrices. Combining with the speed variations, one obtains a 16 sub-models polytopic system.

The controller is computed assuming $25 \%$ contribution of each controller corresponding to one of the vertices. During the simulation, the road friction is reduced by $30 \%$ at the front and the rear wheels during all the simulation duration. Results are shown in Figure 4 for both the nominal case in solid lines and the low friction case in dashed lines. The later are more oscillatory but still acceptable and close to the nominal case. This demonstrates the ability of the controller to handle such situation or reduced road friction.

\section{CONCLUSION}

In this paper, a static output feedback with preview feedforward compensation is considered for ground vehicle
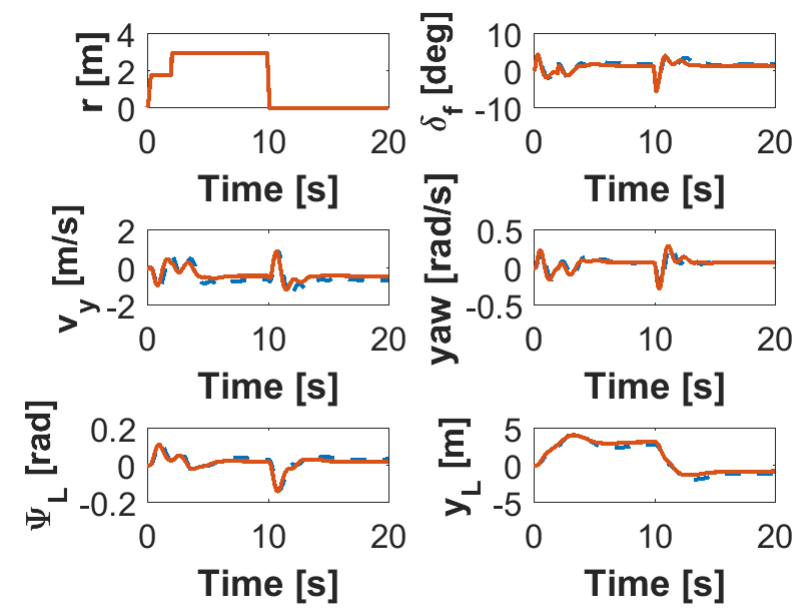

Fig. 4. Lane change maneuver on curved road with reduced friction

autonomous lane change maneuver. This control approach allows a reference signal previewing to be taken into account allowing trajectory tracking during the maneuver. The vehicle model which includes varying vehicle speed is discretized and an error system method allows to obtain a parameter dependent augmented system. A speed dependent static output feedback is then derived using LMI conditions. In its expanded form, it includes a static feedback of the output, the integral of the tracking error and the preview actions. Design parameters allow to have impact on the performance of the system. Simulations results illustrate the advantage of the preview control which allows to adjust the behaviour during the transient phase and to ensure zero tracking error. The formulation of finite future information is particularly convenient in the context of limited preview distance in constrained driving environment.

\section{APPENDIX}

TABLE I

VEHICLE PARAMETERS AND VALUES

\begin{tabular}{|ll|l|}
\hline Parameter & & Value \\
\hline$C_{f}$ & front cornering stiffness & $60000 \mathrm{~N} / \mathrm{rad}$ \\
\hline$C_{r}$ & rear cornering stiffness & $35000 \mathrm{~N} / \mathrm{rad}$ \\
\hline$J$ & vehicle yaw moment of inertia & $2454 \mathrm{~kg} . \mathrm{m}^{2}$ \\
\hline$l_{f}$ & distance form CG to front axle & $1.22 \mathrm{~m}$ \\
\hline$l_{r}$ & distance from CG to rear axle & $1.44 \mathrm{~m}$ \\
\hline$l_{s}$ & lookahead distance & $8 \mathrm{~m}$ \\
\hline$m$ & total mass & $1600 \mathrm{~kg}$ \\
\hline$v_{x}$ & longitudinal velocity & $10-25 \mathrm{~m} / \mathrm{s}$ \\
\hline
\end{tabular}

\section{REFERENCES}

[1] T. Vaa, M. Penttinen, and I. Spyropoulou, "Intelligent transport systems and effects on road traffic accidents: State of the art," IET Intell. Transp. Syst., vol. 1 (2), pp. 8188, Jun. 2007.

[2] J. Nilsson, J. Silvlin, M. Brnnstrm, and E. CoelinghIf, "When, and How to Perform Lane Change Maneuvers on Highways," IEEE Intelligent Transportation Systems Magazine, 68-78, 2016. 
[3] M. Althoff and J. M. Dolan, "Online verification of automated road vehicles using reachability analysis," IEEE Trans. Robot., vol. 30 (4), pp. 903918, 2014.

[4] J. Nilsson, J. Silvlin, M. Brnnstrm, E. Coelingh, and J. Fredriksson, "If, when, and how to perform lane change maneuvers on highways," IEEE Intell. Transp. Syst. Mag., vol. 8 (4), pp. 6878, Winter, 2016.

[5] S. Glaser, B. Vanholme, S. Mammar, D. Gruyer, and L. Nouveliere, " Maneuver-based trajectory planning for highly autonomous vehicles on real road with traffic and driver interaction," IEEE Trans. on Int. Transportation Systems, vol. 11 (3), pp. 589606, 2010.

[6] J. Nilsson, Y. Gao, A. Carvalho, and F. Borrelli, "Manoeuvre generation and control for automated highway driving," in Proc. 19th World Congr. Int. Fed. Autom. Control, Cape Town, South Africa, pp. 63016306, Aug. 2014.

[7] J.M. Maciejowski, "Predictive Control with Constraints," Printice Hall, 2002.

[8] V. L. Bageshwar, W. L. Garrard, and R. Rajamani, "Model predictive control of transitional maneuvers for adaptive cruise control vehicles," IEEE Trans. Veh. Technol., vol. 53 (5), pp. 15731585, 2004.

[9] G. Schildbach and F. Borrelli, "Scenario model predictive control for lane change assistance on highways," in Proc. IEEE Intell. Veh. Symp., Seoul, South Korea, pp. 611616, 2015.

[10] A. Gray, Y. Gao, T. Lin, J. K. Hedrick, H. E. Tseng, and F. Borrelli, "Predictive control for agile semi-autonomous ground vehicles using motion primitives," in Proc. Amer. Control Conf., Montreal, QC, Canada, pp. 42394244, 2012.

[11] R. T. O’brien, P. A. Iglesias, T. J. Urban, ”Lane Change Maneuver via $\mathrm{H}_{\infty}$ Steering Methods," American Control Conference, 1999.

[12] S. Mammar, V. Baghdassarian, D. Koenig, "Robust control and fault detection synthesis with application to tractor-semitrailer automatic steering," IEEE IEEE International Conference on Control Applications, 2000.

[13] J. E. Naranjo, C. Gonzàlez, R. Garca, and T. de Pedro, "Lane-Change Fuzzy Control in Autonomous Vehicles for the Overtaking Maneuver,' IEEE Trans. on Intelligent Transportation Systems, 438-450, 2008.

[14] B. Soualmi, C. Sentouh, J.C. Popieul and S. Debernard, "A shared control driving assistance system : interest of using a driver model in both lane keeping and obstacle avoidance situations," IFAC World Congress, France, 2017.

[15] V. A. Butakov and P. Ioannou, "Personalized driver/vehicle lane change models for ADAS," IEEE Trans. Veh. Technol., vol. 64 (10), pp. 44224431, 2015.

[16] S. J. Anderson, S. C. Peters, T. E. Pilutti, and K. Iagnemma, "An optimal-control-based framework for trajectory planning, threat assessment, and semi-autonomous control of passenger vehicles in hazard avoidance scenarios,” Int. J. Veh. Auton. Syst., vol. 8, pp. 190216, 2010.

[17] G. P. Bevan, H. Gollee, and J. OReilly, 'Trajectory generation for road vehicle obstacle avoidance using convex optimization," J. Automobile Eng., vol. 224 (4), pp. 455473, 2010.

[18] D. Swaroop and S.M. Yoon, "The Design of a Controller for a Following Vehicle in an Emergency Lane Change Maneuver,' Institute of Transportation Studies, Research Reports, Working Papers, Proceedings, 1999.

[19] J. Nilsson, P. Falcone, M. Ali, and J. Sjberg, "Receding horizon maneuver generation for automated highway driving," Control Eng. Pract., vol. 41, pp. 124133, 2015.

[20] J. Nilsson, M. Brännström, E. Coelingh, and J. Fredriksson, "Longitudinal and lateral control for automated lane change maneuvers," in Proc. Amer. Control Conf., Chicago, IL, USA pp. 13991404, Jul. 2015.

[21] J. Nilsson, M. Brännström, J. Fredriksson, and E. Coelingh, ’Longitudinal and lateral control for automated yielding maneuvers," IEEE Trans. Intell. Transp. Syst., vol. 17 (5), pp. 14041414, 2016.

[22] R. Rajamani, Vehicle Dynamics and Control, Mechanical Engineering Series, Springer, 2012.

[23] , Z.L. Li, J.R. Li, "Delay-dependent fuzzy static output feedback control for discrete-time fuzzy stochastic systems with distributed time varying delays," ISA Trans., vol. 51 (6), pp. 702712, 2012.

[24] Z.Y. Feng,L. Xu, J.H. She, et al., ’Optimization of coordinate transformation matrix for $\mathrm{H} \infty$ static-output-feedback control of linear discretetime systems," Asian J. Contr., vol. 17 (11), pp. 1-11, 2015.

[25] P.T. Nam, P.N. Pathirana, H. Trinh, "Convergence within a polyhedron: controller design for time-delay systems with bounded disturbances", IET Control Theory Appl., vol. 9 (6), pp. 905914, 2015.

[26] J.X. Dong, G.H. Yang, "Static output feedback $\mathrm{H}_{\infty}$ control of a class of nonlinear discrete-time systems", Fuzzy Sets Syst, vol. 160 (19), pp. 28442859, 2009.

[27] X.H. Chang, G.H. Yang, "New results on output feedback $\mathrm{H}_{\infty}$ control for linear discrete-time systems," IEEE Trans. Autom. Control, vol. 59, N 5, pp. 1355-1359, 2014.

[28] X.H. Chang, L. Zhang, H.P. Park, "Robust static output feedback $\mathrm{H} \infty$ control for uncertain fuzzy systems," Fuzzy Sets Syst., 273, pp. 87-104, 2015.

[29] J.X. Dong, G.H. Yang, "Robust static output feedback control synthesis for linear continuous systems with polytopic uncertainties,' Automatica, vol. 49 (6), pp. 1821-1829, 2013.

[30] M.J. Cao, F.C. Liao, "Design of an optimal preview controller for linear discrete-time descriptor systems with state delay," Int. J. Syst. Sci., vol. 46 (5), pp. 932-943, 2015.

[31] A. Nasiri, S. Kiong Nguang, A. Swain, et al., "Robust output feedback controller design of discrete-time Takagi-Sugeno fuzzy systems: a nonmonotonic Lyapunov approach," IET Control Theory Appl., vol. 10 (5), pp. 545-553, 2016

[32] F.L. Weng, L.M. Liang, Z.A. Yu, et al., "Online parameter-dependent controller design for electro-hydraulic servo systems driven by doublerod actuators,' J. Comput. Inf. Syst., vol. 8 (22), pp. 9507-9514, 2012.

[33] J. Rubió-Massegú, J.M. Rossell, H.R. Karimi, F. Palacios-Quiñonero, Static output-feedback control under information structure constraints, Automatica, 49, 313-316, 2013.

[34] L. Li, F. Liao, "Parameter-dependent preview control with robust tracking performance," IET Control Theory \& Application, Vol. 11 Iss. 1, pp. 38-46, 2017.

[35] K. Tanaka, H.O. Wang, Fuzzy Control Systems Design and Analysis, Wiley Inc, New York, 2001 